\title{
Chiral molecule adsorption on helical polymers
}

\author{
Maria R. D'Orsogna ${ }^{1}$ and Tom Chou $^{2}$ \\ ${ }^{1}$ Department of Physics \& Astronomy, University of California, Los Angeles, CA 90095-154 and \\ ${ }^{2}$ Department of Biomathematics, University of California, Los Angeles, CA 90095-1766
}

(Dated: November 21, 2018)

\begin{abstract}
We present a lattice model for helicity induction on an optically inactive polymer due to the adsorption of exogenous chiral amine molecules. The system is mapped onto a one-dimensional Ising model characterized by an on-site polymer helicity variable and an amine occupancy one. The equilibrium properties are analyzed at the limit of strong coupling between helicity induction and amine adsorption and that of non-interacting adsorbant molecules. We discuss our results in view of recent experimental results.
\end{abstract}

PACS numbers: 82.35-x, 36.20.-r, 05.50.+q, 61.41.+e

\section{INTRODUCTION}

The chiral properties of macromolecules are of particular importance in determining the nature of their interactions with living material [1]. Most cellular receptor enzymes show preferential binding towards one of the two enantiomers of a helical molecule, the other mirror-image form being either irrelevant or noxious to cellular functioning. Helicity induction and control have also been extensively studied for the purposes of asymmetric synthesis [2], enantiomer separation [3] and other chemosensing, pharmaceutical [4] and material science applications [5].

The helicity of biological macromolecules, such as DNA and proteins, is due to the inherent chirality of the basic components. Besides polymerizing optically active monomers, synthetic chiral polymers may be constructed by asymmetric polymerization of non-chiral molecules using a chiral catalyst, or, as recently reported 6, 7], by complexation of an optically inactive polymer with a chiral amine. The achiral polymer used in Ref. 7, 8] is a polyacetylene (poly-1), a non-helical structure which coils into a helix upon acid-base interaction with right- or left-handed amines and amino-alcohols both in solution and in film. It is believed that the induced helicity is due to the twist irregularity of adjacent double bonds around the single bond of the $-\mathrm{CO}_{2} \mathrm{H}$ functional group of poly- 1 which coils upon interaction with the amine bases. Other achiral polyacetylenes show the same response [8, 9] to chiral amines which may be used to induce a preferential screw sense on achiral polymers. In the experiments of Ref. [7, [8] certain synthetic helical polymers (such as poly-6, poly-7) are also used to separate racemic mixtures, due to their high chiral recognition abilities. These polymers can be used as chiral stationary phases in high performance liquid chromatography (HPLC).

In this paper, we use a lattice model to study helicity induction on a polymer interacting with adsorbed exogenous chiral molecules and for chiral discrimination of racemic compounds. Figure 1 shows the features of the one-dimensional lattice model. Each monomer $i$ of the polymer is associated to a pseudo-spin $\sigma_{i}$ where $\sigma_{i}=\{-1,0,1\}$ represents the induced left $\left(\sigma_{i}=-1\right)$ or

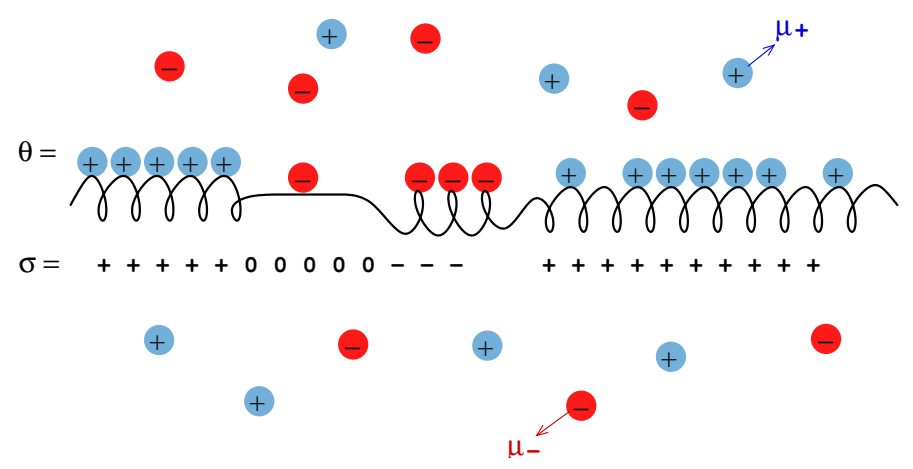

FIG. 1: A schematic of a homo-polymer that can acquire three distinct local conformations. For example, the polymer may acquire right or left-handed helicity, or may remain non-helical. The left region of this figure represents a very inducible polymer with its helicity determined by the binding of the chiral (labeled by + or - ) molecules. The right branch of the polymer represents a locally chiral polymer (which favors say, the + helicity, due to its intrinsic structure) that preferentially adsorbs + molecules. These fixed-helicity polymers have been used to separate + and - chiral molecules in chromatography.

right $\left(\sigma_{i}=+1\right)$ monomer helicity. We also include the possibility for the polymer to be uncoiled, in which case $\sigma_{i}=0$. Site $i$ is also characterized by the chiral molecule occupancy $\theta_{i}$. If the site is unoccupied $\theta_{i}=0$, if it is occupied by a right (left)-handed molecule $\theta_{i}=+1(-1)$. The free energy of the polymer $\Omega$ will be modeled using:

$$
\begin{aligned}
\Omega / k_{B} T= & -J \sum_{i} \sigma_{i} \sigma_{i+1}-\sum_{i}\left(h_{0}+h_{1} \theta_{i}\right) \sigma_{i} \\
& -J_{2} \sum_{i} \theta_{i} \theta_{i+1}-\mu_{+} \sum_{i} \delta_{\theta_{i}, 1}-\mu_{-} \sum_{i} \delta_{\theta_{i},-1}
\end{aligned}
$$

where all parameters appearing on the right-hand side are unitless. In the Ising model defined by Eq. (2), the "magnetic field" $h_{0}$ describes the proclivity of the bare polymer to coil into a left or right helix, and can be interpreted as a net local torsion of the polymer. The $h_{1}$ parameter is the coupling between the chiral amine and 
the polymer. The $J$ and $J_{2}$ parameters are the nearest neighbor energetic coupling for the helicity of the polymer and of the adsorbate respectively. Field force calculations have been used to estimate $J$ and $h_{0}$ for copolymers of enantiomers [14].

The chemical potentials $\mu_{ \pm}$are related to the energy cost for the adsorption of right or left handed amines on the polymer, with the delta functions tracing the number of adsorbed amines. The last two terms of Eq. (2) represent the free energy contribution arising from polymeradsorbate interactions prior to the coiling of the polymer. The chemical potential terms $\mu_{ \pm}$are defined so that $h_{i} \theta_{i} \sigma_{i}$ is the energetic split after adsorption of a molecule with chirality $\theta_{i}$ on site $i$.

For simplicity we assume that the binding sites and the basic polymer helical unit are size-commensurate. Although the microscopic details are more complicated than our model, they should not affect overall equilibrium long wavelength results. Other than chiral interactions, we neglect all other effects that the binding amines might have on the polymer, such as its stiffness although it is well known that binding of small molecules affects the persistence length of polymer chains [10].

Our model is a generalization of a simpler one in which a random chiral copolymer with enantiomeric pendant groups is described in terms of a quenched random-field Ising model 11, 12, 13. In our model $\theta_{i}$ is an unquenched thermodynamic variable related to its chemical potentials $\mu_{ \pm}$. Our model approaches those in Ref. [11, 12, 13] in the limit $\left\{\sigma_{i}=+1,-1\right\},\left\{\theta_{i}=+1,-1\right\}$ and by setting $h_{0}, J_{2}, \mu_{ \pm}=0$.

The equilibrium properties of the model of Eq. (2) can be evaluated through the partition function associated with the nine-dimensional product space $\left|\sigma_{i}, \theta_{i}\right\rangle=$ $|-,-\rangle,|-, 0\rangle,|-,+\rangle,|0,-\rangle,|0,0\rangle,|0,+\rangle,|+,-\rangle$, $|+, 0\rangle,|+,+\rangle$. In the case of a polymer of length $N$ with periodic boundary conditions $\sigma_{1}=\sigma_{N+1}$ and $\theta_{1}=\theta_{N+1}$, the partition function reads:

$$
\Xi=\sum_{\left\{\sigma_{i}\right\}} \sum_{\left\{\theta_{i}\right\}} e^{-\Omega / k_{B} T}=\operatorname{Tr} \mathbf{L}^{N},
$$

where $\mathbf{L}$ is the nine-dimensional transfer matrix derived from $\Omega$. Thermodynamic quantities such as the free energy $F$, the average spin-helicity $\left\langle\sigma_{i}\right\rangle$, the correlation function $\Gamma(\ell)=\left\langle\sigma_{i} \sigma_{i+\ell}\right\rangle-\left\langle\sigma_{i}\right\rangle^{2}$, and the fraction of adsorbed left and right amines $\left\langle n_{\theta= \pm 1}\right\rangle$ are obtained from:

$$
\begin{gathered}
F=-\ln \Xi \\
N\left\langle\sigma_{i}\right\rangle=\left(\frac{\partial \ln \Xi}{\partial h_{0}}\right), \\
\left\langle\sigma_{i} \sigma_{i+\ell}\right\rangle=\frac{\operatorname{Tr}\left\langle\sigma \mathbf{L}^{l} \sigma \mathbf{L}^{N-l}\right\rangle}{\Xi},
\end{gathered}
$$

$$
\text { and }\left\langle n_{\theta= \pm 1}\right\rangle=\left\langle\delta_{\theta_{i}, \pm 1}\right\rangle=\left(\frac{\partial \ln \Xi}{\partial \mu_{ \pm}}\right) \text {. }
$$

The $\sigma$ operator is defined as $\sigma\left| \pm, \theta_{i}\right\rangle= \pm\left| \pm, \theta_{i}\right\rangle$, and $\sigma\left|0, \theta_{i}\right\rangle=0$. Similarly, the $\theta$ operator is $\theta\left|\sigma_{i}, \pm\right\rangle=$ $\pm\left|\sigma_{i}, \pm\right\rangle$, and $\theta\left|\sigma_{i}, 0\right\rangle=0$.

In the $N \rightarrow \infty$ limit Eqs. (46) can be expressed in terms of the eigenvalues of $\mathbf{L}\left(\lambda_{i}=\lambda_{0} \lambda_{1}, \ldots \lambda_{8}\right)$ and their respective ortho-normalized eigenvectors $\left|\psi_{i}\right\rangle$. If the largest eigenvalue is $\lambda_{0}$, with associated eigenvector $\psi_{0}$, then [15]:

$$
\begin{gathered}
\left\langle\sigma_{i}\right\rangle=\frac{1}{\lambda_{0}} \frac{\partial \lambda_{0}}{\partial h_{0}}, \\
\left\langle\sigma_{i} \sigma_{i+\ell}\right\rangle=\frac{\left\langle\psi_{0}\left|\sigma \mathbf{L}^{\ell} \sigma\right| \psi_{0}\right\rangle}{\lambda_{0}^{\ell}} \\
\left\langle n_{\theta= \pm 1}\right\rangle=\left\langle\delta_{\theta_{i}, \pm 1}\right\rangle=\frac{1}{\lambda_{0}} \frac{\partial \lambda_{0}}{\partial \mu_{ \pm}} .
\end{gathered}
$$

The amine helicity correlation function $\left\langle\theta_{i} \theta_{i+\ell}\right\rangle$ and the polymer-amine correlation function $\left\langle\sigma_{i} \theta_{i+\ell}\right\rangle$ can be obtained by substituting the appropriate $\theta$ operator in Eqs. (5) and (8) respectively. The correlation lengths are the same in all cases and we treat only the polymerpolymer correlation function. Numerical estimates of the relevant quantities can be readily computed by diagonalizing the nine-dimensional transfer matrix. However it is revealing to explore certain physically relevant limits analytically. In the following sections, we will consider the experimentally relevant cases of helical polymers used as chiral discriminants, where $\sigma_{i}=+1$ for all $i$ sites, and the transfer matrix is three dimensional. The other physically relevant case is that of chiral amines used to induce helicity on an optically inactive polymer. Complexation with left handed amines yields right handed polymer helicity so we will consider the four-dimensional space given by $\left\{\sigma_{i}=0,+1\right\}$ and $\left\{\theta_{i}=-1,0\right\}$. Both these cases shall be analyzed in the limit of high aminepolymer interaction $\left(\left|h_{1}\right| \gg 1\right)$, and of non-interacting adsorbants $J_{2}=0$. We shall also consider the case of a helical polymer coiling in both screw senses and interacting with both left and right amine isomers. In this case $\left\{\sigma_{i}=-1,+1\right\}$. As above, we shall consider the limits of strong coupling between amine occupancy and polymer helicity $\left(h_{1} \gg 1\right)$, and the limit of non-interacting adsorbant amines, for which $J_{2}=0$.

\section{CHIRAL DISCRIMINATION}

In this section we consider a helical polymer that is chemically fixed to be right-handed $\left(\sigma_{i}=+1\right)$ and analyze 
the mean fraction of left-handed and right-handed chiral amine adsorbates. This case corresponds to the parameters $J \rightarrow \infty$ and $h_{0} \rightarrow \infty$ and we need only consider the reduced basis set $\left|\sigma_{i}, \theta_{i}\right\rangle=|+,-\rangle,|+, 0\rangle,|+,+\rangle$. We wish to calculate the ratio of the two different adsorbate enantiomers: $r=\left\langle n_{\theta=-1}\right\rangle /\left\langle n_{\theta=+1}\right\rangle$. The threedimensional transfer matrix is $\mathbf{L}$ given by:

$$
x_{+}^{-1} \mathbf{L}=\left(\begin{array}{ccc}
\frac{y f_{-}}{z} & 1 & \frac{z f_{+}}{y} \\
\frac{f_{-}}{z} & 1 & z f_{+} \\
\frac{f_{-}}{y z} & 1 & y z f_{+}
\end{array}\right) .
$$

Here, $x_{ \pm} \equiv e^{\left(J \pm h_{0}\right)}, y \equiv e^{J_{2}}, z \equiv e^{h_{1}}$, and $f_{ \pm} \equiv e^{\mu_{ \pm}}$. The largest eigenvalue is readily determined by means of singular perturbation techniques in the limits of strong coupling between polymers and amines, and exactly in the case of non-interacting exogenous molecules.

\section{A. Strong Interaction}

In the case of strong interactions between amines and polymers $\left(h_{1} \gg 1\right.$ or $\left.z \gg 1\right)$, the largest eigenvalue is calculated perturbatively in $z$ to be:

$$
\begin{aligned}
\lambda_{0} x_{+}^{-1}= & f_{+} y z+\frac{1}{y}+\frac{y-1+f_{+} f_{-}}{z y^{3} f_{+}}+ \\
& \frac{2+2 f_{-} f_{+}\left(y^{2}-1\right)-3 y+y^{2}}{f_{+}^{2} y^{5} z^{2}}+\mathcal{O}\left[z^{-3}\right] .
\end{aligned}
$$

Here, $\left\langle n_{\theta=+1}\right\rangle \gg\left\langle n_{\theta=-1}\right\rangle$ because the original choice for the helical polymer $\sigma_{i}=+1$ and positive values for $h_{1}$ favor the adsorption of right-handed amines. The fraction of unoccupied sites vanishes as $1 / z$ and the fraction of left-handed adsorbed amines as $1 / z^{2}$. The concentration of empty sites, and the ratio of adsorbed amines is given by:

$$
\begin{aligned}
\left\langle n_{\theta=0}\right\rangle_{z \rightarrow \infty} & =\frac{1}{f_{+} y^{2} z}-\frac{3-2 y}{f_{+}^{2} y^{4} z^{2}} \\
& \sim e^{-\left(h_{1}+\mu_{+}+2 J_{2}\right)} . \\
{\left[\frac{\left\langle n_{\theta=-1}\right\rangle}{\left\langle n_{\theta=+1}\right\rangle}\right]_{z \rightarrow \infty} } & =\frac{f_{-}}{f_{+} z^{2} y^{4}} \\
& \sim e^{-\left(\mu_{+}-\mu_{-}+2 h_{1}+4 J_{2}\right)} .
\end{aligned}
$$

where $t \equiv e^{h_{0}}, x \equiv e^{J}$, so that $x t=x_{+}$. For the free energy of the bare polymer to be minimum at $\sigma_{i}=0$, the achiral conformation, we assume that $J, h_{0}<0$. To model chiral adsorbates which induce polymer helicity of opposed chirality, we take $h_{1}<0$.

The average adsorbate chirality, $\left\langle\theta_{i}\right\rangle$ (related to the intensity of the circular dichroism spectra of Ref. [6]) is given by $\left\langle n_{\theta=+1}\right\rangle-\left\langle n_{\theta=-1}\right\rangle$. In the opposite limit of negative $h_{1}$ and $\left|h_{1}\right| \gg 1$, the adsorption of left handed amines is favored on a right handed polymer, and a similar calculation yields the same expression for $\lambda_{0}$ as Eq. (10) with the replacements $z \rightarrow z^{-1}, f_{+} \rightarrow f_{-}$. The ratio of right to left-adsorbed amines vanishes as $z^{2}$.

\section{B. Non-interacting Adsorbates}

In the case of non-interacting amines, $\left(J_{2}=0\right.$ or $\left.y=1\right)$ the quantities of interest are calculated exactly by means of Eqs. (7) and (9) with the largest eigenvalue $\lambda_{0}$ :

$$
\begin{aligned}
\lambda_{0} x_{+}^{-1}=\left\langle n_{\theta=0}\right\rangle_{y=1}^{-1} & =\frac{f_{-}+z+f_{+} z^{2}}{z} \\
& \sim e^{-\left(h_{1}-\mu_{-}\right)}+1+e^{\left(h_{1}+\mu_{+}\right)} \\
{\left[\frac{\left\langle n_{\theta=+1}\right\rangle}{\left\langle n_{\theta=-1}\right\rangle}\right]_{y=1} } & =\frac{f_{+} z^{2}}{f_{-}} \\
& \sim e^{+\left(\mu_{+}+2 h_{1}-\mu_{-}\right)} .
\end{aligned}
$$

In this section we consider an achiral polymer which coils into a preferred helicity, right for instance $\left(\sigma_{i}=+1\right)$ upon complexation with external amines of the opposite chirality $\left(\theta_{i}=-1\right)$. This scenario is consistent with the experiments of chiral induction performed with the optically inactive poly- 1 chain in the presence of chiral molecules of specified right or left helicity [a]. In these experiments, complexation with left handed isomers of the chiral molecules induce a right-handed helix on the polymer and vice-versa for right-handed amines. In the basis $\left|\sigma_{i}, \theta_{i}\right\rangle=|0,0\rangle,|0,-\rangle,|+, 0\rangle,|+,-\rangle$, the transfer matrix is four dimensional:

$$
\mathbf{L}=\left(\begin{array}{cccc}
1 & f_{-} & t & f_{-} t z^{-1} \\
1 & f_{-} y & t & f_{-} t y z^{-1} \\
1 & f_{-} & x t & f_{-} x t z^{-1} \\
1 & f_{-} y & x t & f_{-} x t y z^{-1}
\end{array}\right)
$$




\section{A. Strong Interaction}

For highly inducible polymers $\left(\left|h_{1}\right| \gg 1\right.$ or $\left.z \rightarrow 0\right)$, the largest eigenvalue of $\mathbf{L}$ is:

$$
\lambda_{0}=f_{-} x t y z^{-1}+\frac{1+t x^{2}+f_{-} y^{2}}{x y}+\mathcal{O}[z] .
$$

We can compute the fraction of adsorbed amines $\left\langle n_{\theta=-1}\right\rangle$ and the mean induced helicity $\left\langle\sigma_{i}\right\rangle$. Note that since $\sigma_{i}=$ $\{0,+1\}$ the latter is also the fraction of $\left\langle n_{\sigma=+1}\right\rangle$ sites:

$$
\begin{aligned}
\left\langle n_{\theta=-1}\right\rangle_{z \rightarrow 0} & =1-\frac{1+t x^{2}}{f_{-} x^{2} y^{2} t} z \\
& \sim 1-\frac{1+e^{-\left(2 J+h_{0}\right)}}{e^{-\left(h_{1}-\mu_{-}-2 J_{2}\right)}} . \\
\left\langle\sigma_{i}\right\rangle=\left\langle n_{\sigma=+1}\right\rangle_{z \rightarrow 0} & =1-\frac{1+f_{-} y^{2}}{f_{-} x^{2} y^{2} t} z \\
& \sim 1-\frac{1+e^{-\left(2 J_{2}+\mu_{-}\right)}}{e^{-\left(h_{1}-h_{0}-2 J\right)}} .
\end{aligned}
$$

\section{B. Non-interacting Adsorbates}

For non-interacting adsorbates $\left(J_{2}=0\right.$ or $\left.y=1\right)$ the largest eigenvalue for the helical induction matrix $\mathbf{L}$ is:

$$
\lambda_{0}=\frac{1}{2}\left[1+f_{-}+t x+f_{-} t x z^{-1}+\mathcal{C}\right],
$$

where $\mathcal{C}=z^{-1}\left[\left(z+z f_{-}+z t x+f_{-} t x\right)^{2}-\right.$

$$
\left.4 t z(x-1)\left(1+f_{-}\right)\left(z+f_{-}\right)\right]^{1 / 2} \text {. }
$$

The mean helicity and fraction of occupied amine sites are again found explicitly through Eq. (71) and Eq. (9):

$$
\begin{aligned}
\left\langle n_{\theta=-1}\right\rangle_{y=1}= & \frac{f_{-}}{2 \lambda_{0}}\left[1+\frac{t x}{z}+\frac{z+z f_{-}+(t x)^{2}}{z \mathcal{C}}-\right. \\
& \left.\frac{(x-2)\left[2 f_{-} t+f_{-}(t x z)^{2}(1+z)\right]}{z \mathcal{C}}\right], \\
\left\langle n_{\sigma=+1}\right\rangle_{y=1}= & \frac{1}{2}+\frac{z t x-z+(t x-z) f_{-}}{2 z \mathcal{C}} .
\end{aligned}
$$

\section{RANDOM COPOLYMERS}

In this section we analyze the case of a polymer coiling into either screw direction $\sigma_{i}=\{-1,+1\}$ upon interaction with exogenous molecules, $\theta_{i}=\{-1,+1\}$, when no sites are left unoccupied. We shall also assume $J_{2}=0$ throughout the calculation: this case can be viewed as

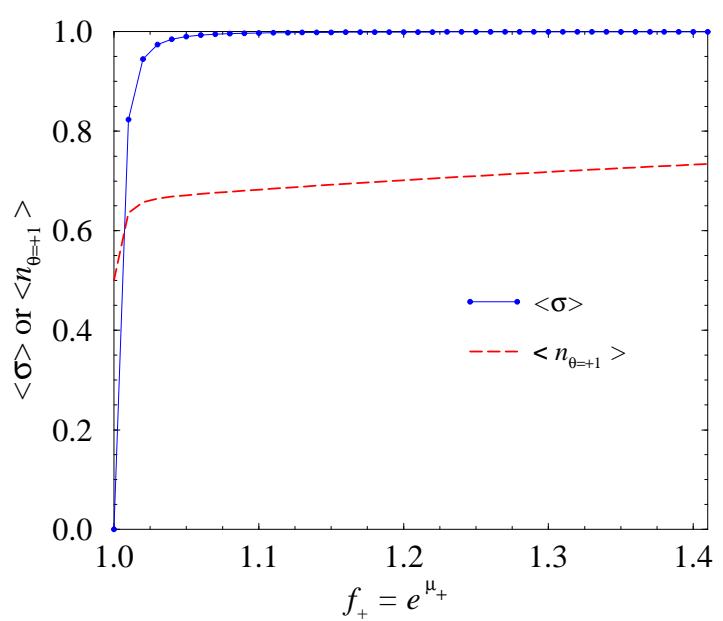

FIG. 2: Mean helicity and amine occupancy as a function of $f_{+}$. The other parameters are fixed at $x_{+}=x_{-}=30$ and $h_{1}=1.4$, as in Ref. [11] and $f_{-}=1$. Note the dramatic increase in $\left\langle\sigma_{i}\right\rangle$ even for values of $f_{+}$slightly greater than unity.

a dynamic version of a model for the the assembly of random copolymers 11] where, instead of a random field $h_{i}=\{+1,-1\}$, assigned with probability $p$ and $1-p$ to each site, we introduce the annealed variable $\theta_{i}$ whose chemical potentials $\mu_{+}$and $\mu_{-}$control the adsorption of each left or right species. In particular, we wish to compare our results with the random field Ising model of [12], due the dynamic nature of the assembly process. The transfer matrix $\mathbf{L}$ is defined in the four dimensional space $\left|\sigma_{i}, \theta_{i}\right\rangle=|-,-\rangle,|-,+\rangle,|+,-\rangle,|+,+\rangle$ :

$$
\mathbf{L}=\left(\begin{array}{cccc}
z f_{-} x_{-} & \frac{f_{+} x_{-}}{z} & \frac{f_{-}}{z x_{-}} & \frac{z f_{+}}{x_{-}} \\
z f_{-} x_{-} & \frac{f_{+} x_{-}}{z} & \frac{f_{-}}{z x_{-}} & \frac{z f_{+}}{x_{-}} \\
\frac{z f_{-}}{x_{+}} & \frac{f_{+}}{z x_{+}} & \frac{f_{-} x_{+}}{z} & z f_{+} x_{+} \\
\frac{z f_{-}}{x_{+}} & \frac{f_{+}}{z x_{+}} & \frac{f_{-} x_{+}}{z} & z f_{+} x_{+}
\end{array}\right)
$$

and its largest eigenvalues are:

$$
\begin{aligned}
\lambda_{0,1}= & \frac{x_{-}\left(f_{+}+z^{2} f_{-}\right)+x_{+}\left(f_{-}+z^{2} f_{+}\right)}{2 z} \pm \frac{1}{2 x_{-} x_{+} z^{2}}(22) \\
& \left\{x_{-} x_{+}\left[x_{-}\left(f_{+}+z^{2} f_{-}\right)+x_{+}\left(f_{-}+z^{2} f_{+}\right)\right]^{2}+\right. \\
& \left.4 x_{+} x_{-} z^{2}\left(1-x_{-}^{2} x_{+}^{2}\right)\left(f_{+}+z^{2} f_{-}\right)\left(f_{-}+z^{2} f_{+}\right)\right\}^{1 / 2} .
\end{aligned}
$$

The mean helicity $\left\langle\sigma_{i}\right\rangle$ and fraction of adsorbed amines $\left\langle n_{\theta=+1}\right\rangle$ can be evaluated by performing the appropriate derivatives and the correlation length is given by 
Eq. (29). Instead of explicitly writing the expressions for these quantities we plot $\left\langle\sigma_{i}\right\rangle,\left\langle n_{\theta=+1}\right\rangle$ as a function of $f_{+}$for the chosen parameters $x_{+}=x_{-}=x=30$, which implies $h_{0}=0$, and $h_{1}=1.4$. We also set the reference energy at $f_{-}=1$, or $\mu_{-}=0$. The values for $x$ and $h_{1}$ correspond to the molecular modeling values used in [12. The physical quantities $\left\langle\sigma_{i}\right\rangle$ and $\left\langle n_{\theta=+1}\right\rangle$ are shown in Figure [2 as a function of $f_{+}$. As in the case of the Ising random field [12], a small increase of the fraction of adsorbed amine $\left\langle n_{\theta=+1}\right\rangle$ for $f_{+} \gtrsim 1$ results in a sharp rise in the optical activity of the sample as determined by $\left\langle\sigma_{i}\right\rangle$, similarly to the experimental results of [16].

\section{CHIRAL ADSORBATES AND POLYMERS}

We now analyze the case of a chiral polymer interacting with a racemic mixture of chiral adsorbates. The transfer matrix $\mathbf{L}$ determined from Eq. (2) in the six-dimensional space defined by $\left|\sigma_{i}, \theta_{i}\right\rangle=$ $|-,-\rangle,|-, 0\rangle,|-,+\rangle,|+,-\rangle,|+, 0\rangle,|+,+\rangle$ is:

$$
\mathbf{L}=\left(\begin{array}{cccccc}
y z f_{-} x_{-} & x_{-} & \frac{f_{+} x_{-}}{y z} & \frac{y f_{-}}{z x_{-}} & \frac{1}{x_{-}} & \frac{z f_{+}}{y x_{-}} \\
z f_{-} x_{-} & x_{-} & \frac{f_{+} x_{-}}{z} & \frac{f_{-}}{z x_{-}} & \frac{1}{x_{-}} & \frac{z f_{+}}{x_{-}} \\
\frac{z f_{-} x_{-}}{y} & x_{-} & \frac{y f_{+} x_{-}}{z} & \frac{f_{-}}{y z x_{-}} & \frac{1}{x_{-}} & \frac{y z f_{+}}{x_{-}} \\
\frac{y z f_{-}}{x_{+}} & \frac{1}{x_{+}} & \frac{f_{+}}{y z x_{+}} & \frac{y f_{-} x_{+}}{z} & x_{+} & \frac{z f_{+} x_{+}}{y} \\
\frac{z f_{-}}{x_{+}} & \frac{1}{x_{+}} & \frac{f_{+}}{z x_{+}} & \frac{f_{-} x_{+}}{z} & x_{+} & z f_{+} x_{+} \\
\frac{z f_{-}}{y x_{+}} & \frac{1}{x_{+}} & \frac{y f_{+}}{z x_{+}} & \frac{f_{-} x_{+}}{y z} & x_{+} & y z f_{+} x_{+}
\end{array}\right)
$$

As above, we shall consider the limits of strong coupling between amine occupancy and polymer helicity $\left(\left|h_{1}\right| \gg k_{B} T\right)$, or $z \rightarrow \infty$, or $z \rightarrow 0$ and the limit of non-interacting adsorbant amines, for which $J_{2}=0$, or $y=1$.

\section{A. High inducibility}

In this section we consider the limit of $\left|h_{1}\right| \gg 1$, corresponding to $z \rightarrow \infty$ or $z \rightarrow 0$. In the first case homochirality between polymer and adsorbates are favored, in the latter opposite screw directions are. Let us assume $z \rightarrow \infty$. Eigenvalues and and eigenvectors are evaluated in terms of $y, p$ and $w$ defined as:

$$
\begin{aligned}
& p=\frac{f_{-} x_{-}}{f_{+} x_{+}}=e^{\left(\mu_{-}-\mu_{+}-2 h_{0}\right)}, \\
& w=x_{+} x_{-}=e^{2 J} .
\end{aligned}
$$

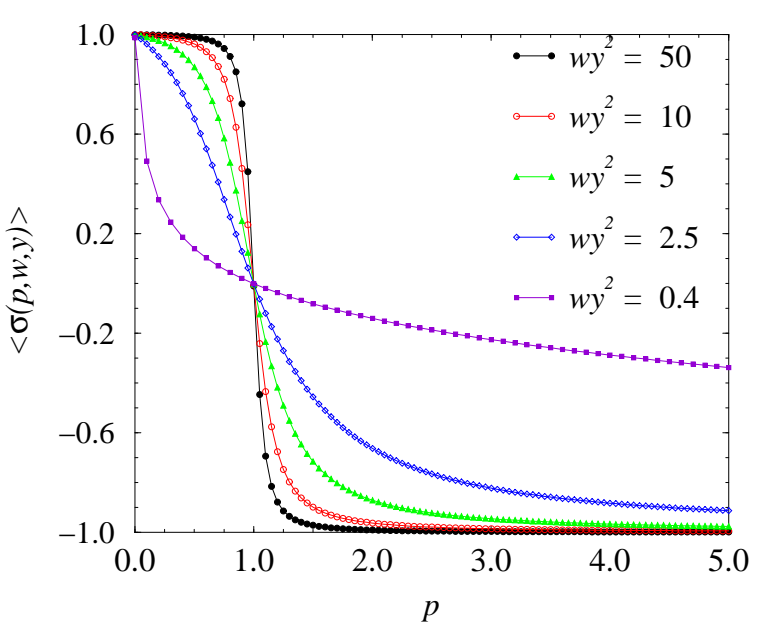

FIG. 3: Mean helicity as a function of $p$ in the case of a polymer with highly inducible chirality $\left(h_{1} \gg 1\right)$. In these curves $w y^{2}=e^{2\left(J+J_{2}\right)}=50,10,5,2.5,0.4$.

A direct calculation for the first two eigenvectors $\lambda_{0,1}$ yields:

$$
\begin{aligned}
\lambda_{0,1} & =\frac{z y f_{+} x_{+}}{2}[(1+p) \pm S(p, w, y)], \\
S(p, w, y) & =\left[(1-p)^{2}+\frac{4 p}{w^{2} y^{4}}\right]^{1 / 2} .
\end{aligned}
$$

The other eigenvectors vanish in the limit $z \rightarrow \infty$ and the mean helicity, from Eq. (7), is:

$$
\left\langle\sigma_{i}\right\rangle=\frac{1-p}{S(p, w, y)} .
$$

Figure [3] shows several $\left\langle\sigma_{i}\right\rangle$ curves for different ferromagnetic and antiferromagnetic values of $w y^{2}$ as functions of $p$. Note that the crossover between positive and negative $\left\langle\sigma_{i}\right\rangle$ values occurs for $p=1$, which corresponds to $\mu_{+}=\mu_{-}-2 h_{0}$. In the limit of high helicity-adsorbate coupling, an overall optically inactive ensemble is reached when the balance between the two amine chemical potentials is offset by the intrinsic torsion $h_{0}$. The sharpness of the transition increases with the ferromagnetic couplings $J$ and $J_{2}$ which tend to induce uniform chiralities on adjacent polymer sites and to adsorb amines with uniform chiralities which, in turn, are strongly coupled to the polymer. The substitution $p \rightarrow p^{-1}$ implies an inversion of the helicity, and under this transformation $\left\langle\sigma_{i}\right\rangle \rightarrow-\left\langle\sigma_{i}\right\rangle$. The correlation function is defined as $\Gamma_{z}(p, w, y, \ell)=\left\langle\sigma_{i} \sigma_{i+\ell}\right\rangle-\left\langle\sigma_{i}\right\rangle^{2}$. The on-site fluctuation is $\Gamma_{z}(p, w, y, \ell=0)=1-\left\langle\sigma_{i}\right\rangle^{2}$ and in the limit of large distances $\ell$, the correlation function $\Gamma_{z}(p, w, x, \ell)$ is determined from Eq. (8) using the fact that $\lambda_{n}=0$ for $n>1$ : 


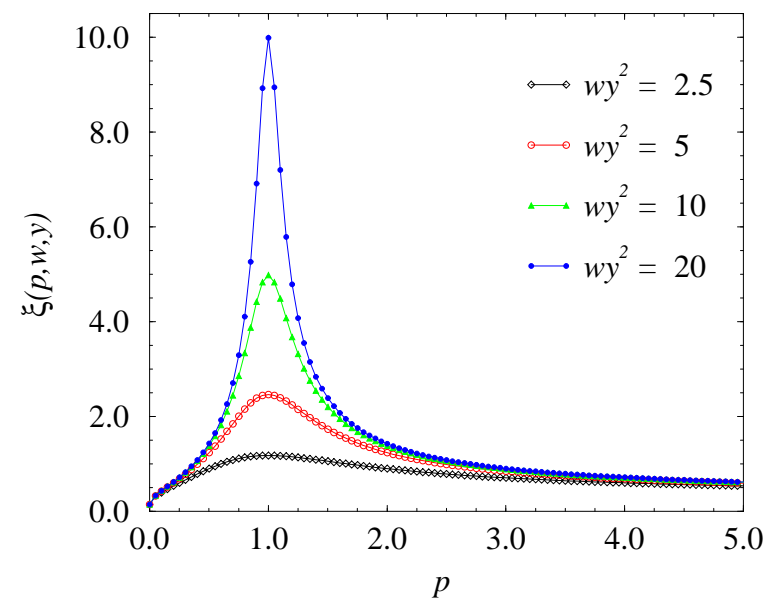

FIG. 4: Correlation length $\xi$ as a function of $p$ in the case of a polymer with highly inducible chirality $\left(h_{1} \gg 1\right)$. In these curves $w y^{2}=e^{2\left(J+J_{2}\right)}=20,10,5,2.5$. Note that at the racemic mixture the correlation length is an increasing function of $w y^{2}$.

$$
\left\langle\sigma_{i} \sigma_{i+\ell}\right\rangle=\left\langle\sigma_{i}\right\rangle^{2}+\left\langle\psi_{0}|\sigma| \psi_{1}\right\rangle\left\langle\psi_{1}|\sigma| \psi_{0}\right\rangle\left(\frac{\lambda_{1}}{\lambda_{0}}\right)^{\ell}
$$

so that:

$$
\begin{aligned}
\Gamma_{z}(p, x, y, \ell \rightarrow \infty) & =D(p, x, y) e^{-\ell / \xi}, \\
\xi^{-1} & =\ln \left(\frac{\lambda_{0}}{\lambda_{1}}\right) .
\end{aligned}
$$

As can be seen from Eq. (27), at the antiferromagnetic condition $J+J_{2}<0$ the second non zero eigenvalue $\lambda_{1}$ is negative, and in the $z \rightarrow \infty$ limit the transfer matrix is no longer positive definite. In calculating the correlation functions we restrict ourselves to the ferromagnetic case of $J+J_{2}>0$. For completeness we estimate the prefactor parameter $D$ using the $\left|\psi_{n}\right\rangle$ eigenvectors found by standard techniques:

$$
\begin{aligned}
D(p, x, y)= & {\left[\frac{\lambda_{0}(1-p+S(p, x, y))}{\lambda_{1} S(p, x, y)}\right]^{2} \frac{\left|\phi_{1}\right|^{2}}{\left|\phi_{0}\right|^{2}} ; } \\
\left|\phi_{0,1}\right|^{2}= & 4 p^{2}\left(1+y^{2}\right)\left(w^{2}+y^{2}\right)+\frac{w^{4} y^{8}}{4} \\
& {[1+p \pm S(p, x, y)]^{2}[1-p \pm S(p, x, y)]^{2}+} \\
& 16 p w^{2} y^{4}[1-p \pm S(p, x, y)]+w^{2} y^{4} \\
& \left(1+y^{2}\right)\left(w^{2}+y^{2}\right)[1-p \pm S(p, x, y)]^{2}+ \\
& w^{2} y^{4}[1+p \pm S(p, x, y)] .
\end{aligned}
$$

Figure 5 shows the fraction of adsorbed right-handed chiral molecules $\left\langle n_{\theta=+1}\right\rangle$ for several values of $w y^{2}$. At the optical inactive condition $p=1$, the fraction of adsorbed amines is $1 / 2$ for each chirality, and all sites are occupied. In this case, both the average amine and polymer helicity vanish and the correlation function depends on the magnitude of $J$ and $J_{2}$. Large values of the latter imply the aggregation of chiral amines of the same sign and islands of homo-helicity both for the polymer and for 
the adsorbed amines. These results are again invariant under the substitution $p \rightarrow p^{-1}$.

\section{B. Non-interacting Adsorbants}

Let us now consider the limit $J_{2}=0(y=1)$, in which the helicity adsorbant amines do not interact with each other, except for exclusion. A direct calculation of the eigenvalues of $\mathbf{L}$ yields:

$$
\begin{aligned}
\lambda_{0,1} & =\frac{F_{+} x_{+}}{2 z}[(1+q) \pm T(q, w)] \text { where } \\
T(q, w) & =\left[(1-q)^{2}+\frac{4 q}{w^{2}}\right]^{1 / 2}, \\
F_{+} & =\left[z^{2} f_{+}+z+f_{-}\right] \text {and } \\
q & =\frac{x_{-}\left[z^{2} f_{-}+z+f_{+}\right]}{x_{+}\left[z^{2} f_{+}+z+f_{-}\right]}=\frac{x_{-} F_{-}}{x_{+} F_{+}} .
\end{aligned}
$$

The parameters $q$ and $p$ of the previous section are related by $\lim _{z \rightarrow \infty} q=p$, and the definition of $w$ is the same as previously defined, $w=e^{2 J}$. All other eigenvalues, $\lambda_{n>1}$ are zero. The mean helicity computed from Eq. (7) is now:

$$
\left\langle\sigma_{i}\right\rangle=\frac{1-q}{T(q, w)},
$$

which has the same form of Eq. (27) upon substitution of $p \rightarrow q$ and $y=1$. In the case of non-interacting amines therefore, the non-optically active condition is $q=1$ or, if we define $2 \mu=\mu_{+}+\mu_{-}$and $2 \Delta \mu=\mu_{+}-\mu_{-}$:

$$
e^{2 h_{0}}=\frac{2+e^{\mu} \cosh \left[\Delta \mu+h_{1}\right]}{2+e^{\mu} \cosh \left[\Delta \mu-h_{1}\right]}
$$

In the non-interacting adsorbant limit, the correlation function $\Gamma_{y=1}(q, w, \ell)$ can be evaluated through Eq. (8) and it is simply Eq.29 with that calculated in the strongly interacting limit provided $p \rightarrow q$ and $y=1$ :

$$
\Gamma_{y=1}(q, w, \ell)=\Gamma_{z}(p, w, y=1, \ell)
$$

The adsorbed amine concentrations $\left\langle n_{\theta= \pm 1}\right\rangle$ do not sum to unity and unoccupied sites exist. Eq. (9) yields:

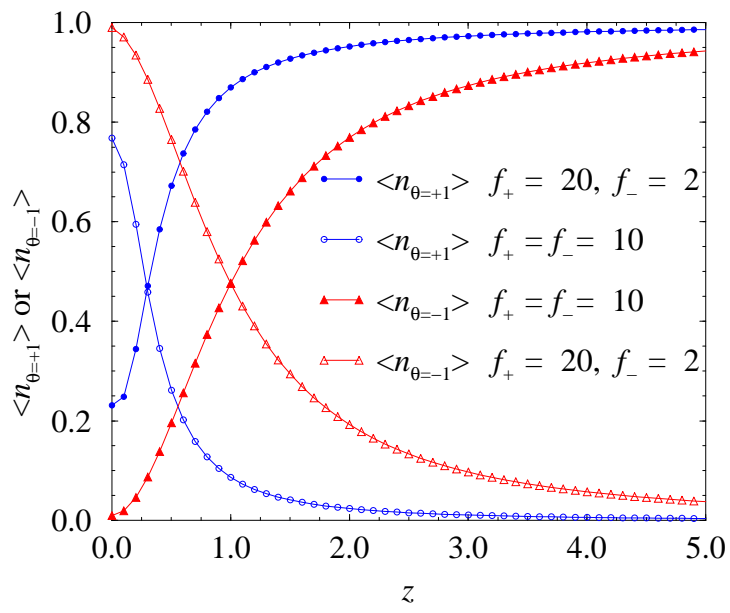

FIG. 6: Fraction of adsorbed amines for several values of $f_{ \pm}$ at $h_{0}=3, w=2$ as a function of $z$. In these plots the dashed curves correspond to the $\left\langle n_{\theta=+1}\right\rangle$; the upper one is at $f_{+}=20, f_{-}=2$, and the lower one at $f_{+}=10, f_{-}=10$. The solid curves correspond to $\left\langle n_{\theta=-1}\right\rangle$; the upper one is at $f_{+}=10, f_{-}=10$ and the lower one at $f_{+}=20, f_{-}=2$.

$$
\begin{aligned}
\left\langle n_{\theta=+1}\right\rangle & =\frac{f_{+} z^{2}}{F_{+}}+\mathcal{G} \frac{q f_{+}}{F_{+} F_{-}}\left(F_{+}-z^{2} F_{-}\right), \\
\left\langle n_{\theta=-1}\right\rangle & =\frac{f_{-}}{F_{+}}+\mathcal{G} \frac{q f_{-}}{F_{+} F_{-}}\left(F_{+} z^{2}-F_{-}\right), \\
\text {where } \mathcal{G} & =\frac{T(q, w)-1+q+\frac{2}{w^{2}}}{T(q, w)[T(q, w)+1+q]} .
\end{aligned}
$$

Several limits are possible for $\left\langle n_{\theta=0}\right\rangle=1-\left(\left\langle n_{\theta=+1}\right\rangle+\right.$ $\left.\left\langle n_{\theta=-1}\right\rangle\right)$ :

$$
\begin{aligned}
& \left\langle n_{\theta=0}\right\rangle=1-\frac{z}{f z^{2}+z+1}, \quad \text { when } f_{+}=f_{-} \\
& \left\langle n_{\theta=0}\right\rangle=1-\frac{z}{f_{+}+f_{-}+1}, \quad \text { when } z=1 \\
& \left\langle n_{\theta=0}\right\rangle=1-\frac{z \cosh \left(h_{0}\right)}{e^{h_{0}} F_{+}}, \quad \text { when } \quad q=1 .
\end{aligned}
$$

\section{CONCLUSIONS}

We have constructed and studied in detail a generalized lattice theory to model interactions between chiral molecules and polymer chains which may or may not be inherently helical. One-dimensional Ising models used to describe chiral systems have been presented in the literature 11, 12, 13] both for ordered and quenched helical polymers. Here, we have introduced the possibility of 
interactions with external amines, incorporated as unquenched thermodynamic variables, as well as the possibility for the polymer to stay uncoiled, $\sigma_{i}=0$. In particular, for a racemic compound interacting with a helical polymer, we derived the ratio of the two adsorbed enantiomer species: the results obtained in Eqs. (11), (14), and (14) can be applied to experimental results to estimate microscopic parameters such as the chemical potentials $\mu_{ \pm}$, the amine coupling $J_{2}$, or the polymer-amine interaction $h_{1}$. Several HPLC experiments estimate the capacity and separation factors (related to the retention times) of chiral amines interacting with chiral poly- 6 and poly-7 used as a molecular sieve [6]. However, the retention times arise from the adsorption and desorption kinetics of the amines as they interact with the stationary phase polymer. The thermodynamic parameters $\left(h_{1}\right.$, $\mu_{ \pm}$etc.) are equilibrium properties and by themselves are insufficient to determine the kinetic capacity factors. However, if the activation barriers for the amine-polymer interactions can be independently determined, capacity factors can then be estimated.

The peak intensity of circular dichroism spectra of optically inactive polymers interacting with chiral molecules can be directly related to the mean induced helicity through Eq. (16). Peak intensity (the mean induced helicity) increases with larger amine size, suggesting that larger exogenous molecules result in higher amine-amine interaction $J_{2}$. This trend is also evident from Eq. (16). Our model does not consider the energetic cost to create a left or right handed helicity domain in an uncoiled region where $\sigma_{i}=0$. An additional free energy term of the form $J_{1}\left[\sigma_{i}^{2}+\sigma_{i} \sigma_{i+1}\left(\sigma_{i}+\sigma_{i+1}\right)+\sigma_{i+1}^{2}\right]$ would be needed to incorporate such effect.

We thank Prof. J. Rudnick and Z.G. Wang for helpful discussions and we acknowledge support from the National Science Foundation through grant DMS-0206733. TC is also grateful to the Japan Society for the Promotion of Science and the National Academy of Sciences for the opportunities provided during the Fourth Annual Symposium on Japanese-American Frontiers of Science which motivated this work.
[1] Chirality and Biological Activity, Edited by B. Holmstedt, H. Frank and B. Testa, (New York, Liss, 1990).

[2] Chiral auxiliaries and ligands in asymmetric synthesis, J. Seyden-Penne, (New York, Wiley, 1995).

[3] Y. Okamoto and E. Yashima, Angew. Chem. Int. Ed. Eng., 37, 1020 (1998).

[4] B. Waldeck, Chirality, 5, 350 (1993).

[5] J. R. Koe, M. Fujiki and H. Nakashima, J. Am. Chem. Soc., 121, 9734 (1999).

[6] E. Yashima, Anal. Sci., 18, 3 (2002).

[7] E. Yashima, T. Matsushima and Y. Okamoto, J. Am. Chem. Soc., 117, 11596 (1995).

[8] E. Yashima, T. Matsushima and Y. Okamoto, J. Am. Chem. Soc., 119, 6345 (1997).

[9] Y. Ashida, T. Sato, K. Morino, K. Maeda, Y. Okamoto and E. Yashima, Macromol., 36 (2003).
[10] H. Diamant and D. Andelman, Phys. Rev. E, 61, 6740 PartB (2000).

[11] M. M. Green, J. W. Park, T. Sato, A. Teramoto, S. Lifson, R. L. Selinger and J. V. Selinger, Angew. Chem. Ed. Int. Eng., 38, 3138 (1999).

[12] R. L. Selinger and J. V. Selinger, Phys. Rev. Lett. 76, 58 (1996).

[13] R. L. Selinger and J. V. Selinger, Phys. Rev. E 55, 1728 (1997).

[14] S. Lifson, C. E. Felder, M.M. Green, Macromol., 25, 4142 (1992).

[15] Statistical Field Theory, G. Parisi, Frontiers in Physics Lecture Notes Series, (Addison-Wesley, 1988)

[16] M. M. Green at al, Science, 268, 1860 (1995). 